International Journal of Engineering \& Technology, $7(4.12)(2018) 20-25$
International Journal of Engineering \& Technology
SPC
Website: www.sciencepubco.com/index.php/IJET
Research paper

\title{
A Survey of Routing Protocols in Wireless Sensor Networks
}

\author{
Navneet Kaur ${ }^{1 *}$, Dr. Sahil Verma ${ }^{2}$, Dr. Kavita ${ }^{3}$ \\ ${ }^{1}$ Research scholar, Lovely Professional University, Phagwara, India \\ ${ }^{2}$ Assistant Professor, Lovely Professional University, Phagwara, India \\ ${ }^{3}$ Assistant Professor, Lovely Professional University, Phagwara, India \\ *Corresponding author E-mail: sahil.21915@lpu.co.in
}

\begin{abstract}
Wireless Sensor Networks(WSNs) comprise sensor nodes which find applications in a wide variety of fields such as medical, wildlife, security, environment, industry. A network communication is initialized and accomplished with the aid of routing protocols. A routing protocol is a set of rules which govern the routing phenomenon. WSNs protocols for the purpose of routing have been the ubiquitous option of the researchers in the recent years due to their exorbitant scope of improvement. The objective of a routing protocol is to inquest for a relevant route amidst sender and receiver to accomplish successful transmission at the destination .Dissipation of energy and lengthening the duration of the network have always been one of the major points of research gaps. As the nodes in WSNs in are battery operated, so they can only use restricted energy to proceed with the communication and transmission operation. To cope up with this, a number of researchers have come up with developments in the field of energy efficacy and optimizations in WSNs routing protocols. A reify summarization of some protocols for routing purposes has been manifested in this paper.
\end{abstract}

Keywords: WSNs, LEACH, PEGASIS, SPIN.

\section{Introduction}

WSNs are composed of sensor nodes disposed in a specific area. A sensor is nothing but an equipment or device that executes the collection and transmission of messages among other sensors. A sensor in a WSN is composed of four vital elements. These include sensing module, a processing module, a transmission module, and a power module. Each one of these has a function of its own. A sensing unit assembles the information, the processing unit consists of microprocessor to process the information, the transmission unit transmits the information, and a power unit is responsible for the power supply. The sensors sense the information, aggregate or fuse the data and the data is successfully achieved by the base station. The base station or gateway is the intended destination for every data. Fig 1 depicts the scenario of WSNs. The figure depicts a group of sensor nodes along with the gateway or base station. To accomplish a complete communication and transmission to intended destination, a routing protocol is used. To devise a routing protocol that can overcome the problems energy, security, communication, connectivity in WSNs is not a simple task. There are several categories of routing protocols in WSNs which have been elaborated in the next section.

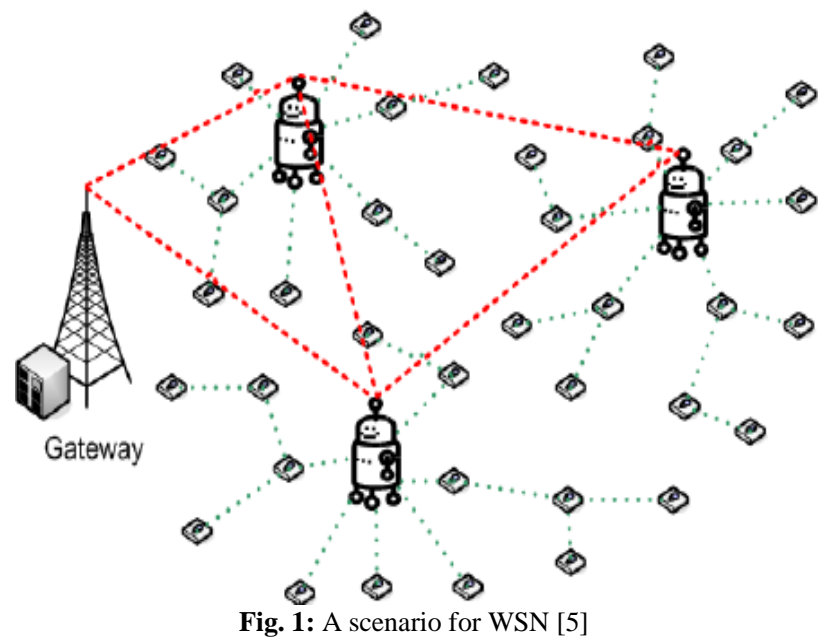

1.1 Categories of routing protocols in WSNs

Routing has always been one of the trending issues in the recent years of WSNs. Routing refers to the process of communication and transmission of data among the nodes. Routing in WSNs suffers a major challenge of energy consumption.

The different categories for protocols in routing for WSNs have been displayed as:

a. Hierarchical routing protocol - From the name itself is clear that why they are known as hierarchical protocols. They involve hierarchical configuration of sensors for the purpose of routing. A particular type of network configuration is followed here. Configuration can be done according to groups that may comprise a predefined node count and these nodes belong to only that particular group. The sensors are required 
to work at different levels of functionality. Depending on the functionality, it can comprise a number of levels. Cluster based routing is the most common configuration in this category of routing.LEACH and PEGASIS are major hierarchical protocols for routing that are based on clustering.

b. Data centric protocol-These are interest based protocols. Interests are disseminated throughout the network. Here, queries are disseminated throughout the network based on an attribute regarding a phenomenon.Here, the nodes broadcast information based on some interest. And the nodes which find that the interest is matching their own, theycan demand to have that interesting information. Hence, this works in form of publish and subscribe model. Here, publish depicts advertising or disseminating the interesting information by the nodes while subscribe depicts demanding that interesting information by nodes to which it was advertised. SPIN Directed Diffusion, and rumor routing follow this kind of approach. The node does notneed to demand the information in which it is interested from a specific node. The node with which that interesting information is available can provide it.

c. Location/Geographical/ Position based routing protocol - In this category, the position of nodes playvery significant part. It's of paramount in this type of routing. The importance of content is more than that of the identity. The nodes can deliver queries related to a specific fact or situation. This approach is better than the conventional approaches for those situations where the identity of the node is not significant to routing as compared to geographical position of the node. It is better because it needs low power dissipation. In conventional routing protocols, the protocol is required to be aware of the full network topology including the identity of the nodes, the routes to be followed for routing fashion, the sources and the sinks. Hence, overhead increases in conventional protocols. However, this is not the case with geographical routing schemes by the cause they don't need to preserve the thorough information of the network.Eg, GEAR.

d. Flat based routing protocol -It is also called as peer based routing protocol. The concept is to call it as a flat based or peer based is that all nodes work on the same level as they have same kind of purpose. No specific type of network configuration is deployed here. In other words, each node will aid in the purpose of routing to transfer information. The routing protocols falling under this category comprise flooding and gossiping. Fig 2 depicts the different categories of protocols for routing in WSNs.

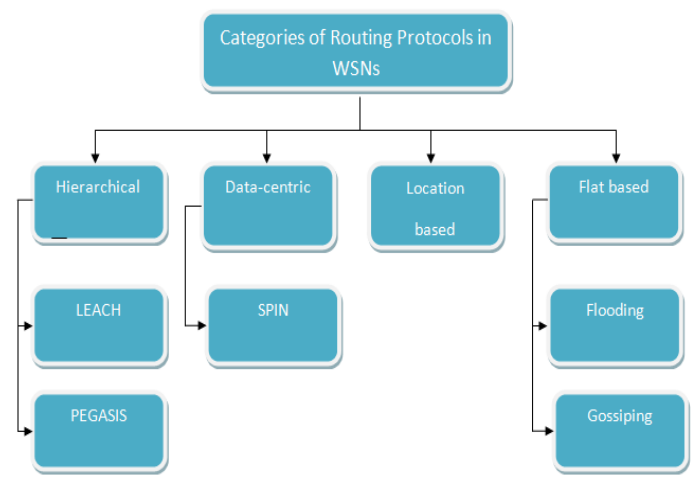

Fig. 2: Categories of routing protocols in WSN

\section{Related Work}

S. Lindesey, and C. S. Raghavendra [3] proposed PEGASIS: Power-Efficient Gathering in Sensor Information Systems.One of the most talked about drawbacks of WSN is undoubtedly restricted power of the battery of nodes and energy consumption. It is very critical to operate the whole network in an efficient manner in such a situation. In the early years, LEACH protocol was proposed so as for solving this issue. In LEACH, network is disposed in the form of clusters, a cluster head $(\mathrm{CH})$ is appointed from each and every cluster. The nodes in every cluster convey the data to their respective $\mathrm{CHs}$, means cluster heads are responsible for aggregation or fusion of the data. This way redundancy is also removed. Finally, the complete sensed information is achieved at the base station or sink. The proposed protocol, known as PEGASIS, follows an approach based on chain (greedy algorithm). This protocol is an improvement over the previous one (LEACH) in which communication is accomplished only with the nearest neighbor and then final transmission to the base station. Unlike LEACH, in PEGASIS, aggregation of data is accomplished at each and every node apart from the node at the end in the chain. PEGASIS saves more energy as compared to LEACH. $100 \%$ to $300 \%$ of better performance was found in case of PEGASIS and compared to LEACH. Performance was found better when $1 \%, 20 \%, 50 \%$, and $100 \%$ of nodes die.

J. Hong, J. Kook, S. Lee, D. Kwon, and S. Yi [6] proposed TLEACH. In this improved protocol, role of residual or remaining energy threshold is to minimize the count of selection of $\mathrm{CH}$, or in other words the $\mathrm{CH}$ count must be limited. More the $\mathrm{CH}$ count, more will be the energy dissipated, hence this scheme was proposed. This was mainly introduced to enhance the duration of the network. In the simulation results, T-LEACH performed better than LEACH on the basis of duration of the network and balancing energy.

H. M. Abdulsalam, and L. K. Kamel[7] proposed W-LEACH: Weighted Low Energy Adaptive Clustering Hierarchy Aggregation Algorithm for Data Streams in Wireless Sensor Networks. This improved protocol was introduced for homogenous as well as heterogenous networks for improving network duration. Similar to LEACH, this protocol also comprises two phases - setup and steady state. Weight value is responsible for choosing the cluster head and a maximum of $\mathrm{p} \%$ working sensors are appointed as $\mathrm{CH}$ s ignoring whether that sensor node was appointed as $\mathrm{CH}$ in the previous rounds or not. Only $\mathrm{x} \%$ of the sensor nodes are chosen in every cluster head so as to transmit data to their respective $\mathrm{CHs}$. These sensorare also chosen on the basis of their weights. The factors on which the weight depends are remaining energy $e_{i}$ and density $d_{i}$. Density factor deals with non-uniform parameters. Density and remaining energy are directly proportional to the number of nodes. The performance of W-LEACH was compared with LEACH on the basis of death of first node, last node, and average sensor duration, number of alive nodes and remaining energy. W-LEACH outperformed LEACH in all these parameters.

Z. Rehena, S. Roy, and N. Mukherjee [8] proposed SPIN with modification for WSNs. The original SPIN protocol consists of ADV, REQ, and DATA phases. A new phase is introduced in the modified protocol and that is known as distance discovery. This distance discovery phase is based upon the hop value. A startup packet that consist of type of message, nodeID, and hop is broadcast by the sink node. Whenever this startup packet is received by a node, it stores the hop value, increments it by one and then retransmits to the other nodes with the incremented hop value. This goes on until the startup packet is received by all sensors. The negotiation and data transmission phases are similar to SPIN-BC protocol.If node's hop is less than the hop that is received then an REQ message will be send by the receiver node to the sender one to have the current data. The actual data will be then send to the requesting node. The performance was compared with SPIN-BC on the basis of packet transmission and dissipation of energy. Performance was found to be better.

Q. Liao, and H. Zhu [10] proposed a protocol optimizes the election of the $\mathrm{CH}$ on the basis of threshold. It uses a cost function for the 
optimal selection of cluster head along with the position of the node and current energy of the node. So, nodes with high amount of energy have better chances than the other ones to get chosen as the $\mathrm{CH}$ selection. This protocol also considers the distance between the sensor and BS. If distance is not too much then there is no need of forming $\mathrm{CHs}$ and directly the sensor node can send the data to the BS.

Z. Beiranvand, A. Patooghy, and M. Fazeli [11] proposed ILEACH. This improved protocol recognizes parameters such as remaining energy of node that is to be chosen as the $\mathrm{CH}$, degree or number of neighboring nodes or location of nodes, as well as distance between $\mathrm{CH}$ and sink. It also consists of the same phases with modification. The phases include $\mathrm{CH}$ selection, cluster development, and the data transmission phase. This proposed protocol was compared with other protocols such as LEACH, and DBS. It was found to outperform other two protocols on the basis of duration of WSN and average energy consumption.

S. E. Khediri, N. Nasri, A. Wei, and A. Kachouri [12]proposed a scheme known as O-LEACH is a variation of the LEACH protocol. The remaining energy is calculated before hand for the node that is required to be selected as the leader. Here, that sensor is selected as $\mathrm{CH}$ which is has the energy value $10 \%$ greater than the residual energy at every other node. Then afterwards the process is same as the LEACH protocol. For the next rounds if the value of energy is less, then the LEACH protocol will be followed. The simulation was carried out in Matlab (version 7.0) using static networks and the results were compared with LEACH and LEACH-C. The parameters taken were number of alive nodes (on 300 nodes and position of the base station) and energy dissipation (on 100 and 300 nodes).

J.Grover, B. Noble, and I.N. Sneddon [13] proposed a reliable SPIN in WSNs. In the original SPIN protocol, there wasn't any guarantee that whether the data has reached its destination or not. As WSNs, focus on reliable data delivery, a new phase was added to SPIN known as ACK(Acknowledgement) from the sink to the nodes. In this way, data delivery will be improved. The performance was compared with basic SPIN on the basis ofrouting overhead, throughput, average balance energy, and packet delivery ratio (PDR), and performance was found to be better as compared to basic SPIN.

A. K. Mishra, R. U. Rahman, R. Bharadwaj, and R. Sharma [14] proposed an enhancement of original PEGASIS. The proposed protocol known as PEGASIS-INL was introduced as a modification over the original protocol. In original PEGASIS, the leader is elected within the chain so as to aggregate and convey the final data to the base station. But the protocol can be disadvantageous if the leader that is selected is at a greater distance from the base station. Chain formation modification was done in PEGASIS-INL so as to improve network duration. Only that node is elected as the leader which is nearest to $\mathrm{BS}$ or in other words which is in the strong radio range of the base station. Similar to PEGASIS, it has the phases such as leader selection, chain formation, data aggregation and data transmission. In the leader selection phase, the base station transmits an echo to the nodes in the network. If the threshold value is lesser than this RSSI value, then it is becomes a candidate to be elected as the leader of the chain. Overlapped chain formation is use here. Here, every node has the shortest route to each and every candidate in their route-vector table. This route vector helps in sending the data to the leader. Token passing is not a part of PEGASIS-INL, unlike PEGASIS. Data fused at every node and finally the data is transmitted to BS. The performance of PEGASISINL was compared with PEGASIS average energy consumed, average remaining energy, number of alive nodes, number of dead nodes. PEAGASIS-INL outperformed PEGASIS in all these parameters.
A. Tripathi, N. Yadav, and R. Dadhich [15] implemented SPIN in clustered environment. This protocol is a variation of the original SPIN. The proposed scheme uses the clustering algorithm with SPIN protocol. The three phases in this scheme are cluster head generation, negotiation and transmission. The cluster head is selected within $50 \mathrm{~m}$ of the distance. After each round, the rotation of $\mathrm{CH}$ role is decided by the negotiation phase. The $\mathrm{CH}$ is rotated on the basis of sensor's remaining energy. Conclusively, data is conveyed to $\mathrm{BS}$ by $\mathrm{CH}$. The proposed scheme was compared to LEACH on the basis of duration of the network, energy depletion and $\mathrm{CH}$ formation. The proposed scheme outperformed $\mathrm{LEACH}$ protocol.

K. Khan, M. Sajid, S. Mahmood, Z. A. Khan, U. Qasim, and N. Javaid [16] proposed $\mathrm{LEACH}^{2}$. In this paper, an enhanced technique over the original LEACH is implemented in which the network has been studied on the basis of dividing the network into four regions and performance has been studied on the basis of one, two and three sinks. Three sinks were deployed at center, left and right of the field and from each of these regions a cluster head was selected. Also the nodes can transmit data directly to BS as distance between ordinary sensor and BS is lesser than the distance between $\mathrm{CH}$ and the ordinary sensor. The procedures such as the selection of cluster head formation, data transmission is same as the original LEACH protocol. The performance was studied on the basis of parameters such as dead (non-operational) nodes, alive (operational) nodes, throughput, average residual energy and average delay. It was found out that LEACH protocol performs better with all the parameters when three sinks are deployed in the network. It improves the stability of the network, its lifetime and throughput.

A. Razaque, M. Abdulgader, and C. Joshi [18] proposed P-LEACH. The existing protocols were having various drawbacks including power consumption and energy as the most important ones. Protocols such as LEACH and PEGASIS were having their own individual drawbacks. LEACH had cluster-based architecture limitations but has the advantage of dynamicity. PEGASIS removes this limitation of LEACH lacks the feature of dynamicity. So, a combination of both these protocols was proposed called as PEGASIS-LEACH or P-LEACH. The improved protocol overcomes the limitation of both the original protocols. It is an optimization of the two protocols that give result to cluster based chain protocol. In this improved protocol, the network is disposed intovarious clusters. A CH is elected within every cluster. The data is aggregated and transmitted through these cluster heads thus forming a chain and conclusively the data is conveyed to BS. And $\mathrm{CH}$ that is in close proximity to BS is chosen as the leader of chain and is assigned the responsibility to send the data directly to the base station. The performance of P-LEACH was compared with LEACH and PEGASIS on basis of dead nodes count and average energy of nodes. Performance was found to be better on the basis of improving the network duration, depletion of energy and number of dead sensors. P-LEACH had the minimal number of dead sensors, and also had the maximum average sensor energy.

I. Boulhares, and M. Omari [21] proposed a hybrid scheme of PEGASIS with LEACH-1R protocols in WSNs. This protocol is an enhancement over LEACH-1R and PEGASIS protocol. It is an optimal technique over the drawbacks of chain supported PEGASIS and cluster supported LEACH. It is a combination of both cluster based and chain based protocols. Cluster heads are chosen according to the LEACH-1R rule and after the cluster heads are elected, a chain is formed among these cluster heads using PEGASIS protocol (greedy algorithm rule). This improved protocol is known as LEACH-1R PEGASIS. Then leader is elected from the nodes forming the chain. The node which is nearest to the sink is chosen as the leader. This protocol is called as the LEACH-1R inter PEGASIS. The performance of LEACH-1R PEGASIS was compared to LEACH and PEGASIS on the basis of alive or working sensors count and data delivery to base station. From simulation results, it was found that that the suggested protocol 
performed better than PEGASIS as it uses the advantage of clustering. This protocol improves duration of the network and also, scalability and tackles with long transmission delay. As of future scope, according to authors a new algorithm can be introduced in order to construct the chain rather than using the greedy algorithm approach.

M. Arioua, Y. Assari, I. Ez-zazi, and A. Oualkadi[22] proposed a combination of LEACH protocol and MTE protocol. As transmission based on single hop between sensor and $\mathrm{CH}$ reduces the performance of the network, so multi hop communication approach has been fused with LEACH protocol in order to optimize the communication and the transmission process and get an optimized result in form of extended network duration. Here, the intermediate nodes are used for transmitting data to $\mathrm{CH}$. The sensor node forwards the data to the successor in closest proximity to it The successor is required to aggregate and compress the data before forwarding it to its next successor till it reaches the $\mathrm{CH}$. The $\mathrm{CHs}$ then receive and cumulate the data, remove redundancies and finally transmit to the base station. This combined approach was compared with both LEACH and MTE protocols in terms of total system energyand operational sensors count. The results were found to be better as the proposed scheme helped in the optimization of energy.

R. Dutta, and S. Gupta [23] proposed energy aware modified PEGASIS. In this modified version of protocol, the nodes communicate with the nearest nodes. Parameters such as distance and unconsumed or remaining energy were taken into consideration for this protocol. The performance was compared on the basis of energy depletion and performance was found to be better.

A. Krishnakumar, and V. Anuratha[24] proposed an energyefficient $\mathrm{CH}$ selection of LEACH Protocol in WSNs. The protocol works according to the two parameters which are: $\mathrm{CH}$ and other sensors' in between distance, degree or number of neighboring sensors. Hence, helps in the enhancement of the threshold value so as to efficiently form the cluster heads. Along with the probability function, as in case of original LEACH protocol, this protocol also uses two function called score function( $\mathrm{SnFn}$ ) which are based on the above two parameters for the proposed protocol. This protocol outperformed the leach protocol by $65 \%$ based on working sensors count, and average leftover energy on the basis ofdistance between node and neighbors. Though the proposed protocol was found to improve the network lifetime and scalability, it doesn't suitssmallsized networks.

\section{Routing Protocols in WSNs}

\subsection{LEACH (Low-Energy Adaptive Clustering Hierarchy)}

The sensor network is split into several clusters with each one of them having a cluster head $(\mathrm{CH})$. The one having the maximum remaining energy performs the role of a cluster head. Generally, it comprises two phases - setup, and steady-state. The former phase comprises the selection of $\mathrm{CH}$ based on the threshold value $\mathrm{T}(\mathrm{n})$. If the arbitrary number generated by any node is less than $T(n)$, then that node will be chosen as the $\mathrm{CH}$. T(n) is given by

$$
T(n)=\{0 \quad \text { if } n \notin G
$$$$
\frac{P}{1-P\left(r \bmod \left(\frac{1}{P}\right)\right)} \forall \mathrm{n} \in \mathrm{G}
$$

Where $\mathrm{P}$ represents $\mathrm{CH}$ probability, $\mathrm{r}$ represents ongoing round, $\mathrm{G}$ represents those nodes which were not selected as $\mathrm{CH}$ during the last 1/P rounds. The $\mathrm{CH}$ then disseminates this information across the network in order to let the other sensor nodes to know about its new role. The sensor nodes choose on cluster of which it can become a member. The factor involved in this case is the received signal strength. Then they inform the $\mathrm{CH}$ that they want to join that cluster. Once the $\mathrm{CH}$ is chosen, it assigns a TDMA schedule and a CDMA slot to every other sensor node so that they can transmit the data in that particular TDMA schedule and avoid collisions. Collisions are avoided with the help of CDMA code. The latter phase, known as the steady state phase consists of gathering, fusion and transmission of data. In this phase, the sensors will gather the packets and transmit them to their respective $\mathrm{CHs}$ in their allotted TDMA schedule and CDMA code. The $\mathrm{CH}$ finally cumulative the collected data eliminates redundancies and transmits it to the base station. The responsibility of $\mathrm{CH}$ keeps on rotating giving a view consistent energy dissipation of energy in the network. The $\mathrm{CH}$ does this so as to reduce unnecessary transmissions count because of the energy restraints of the network. The objective of this protocol is to reduce energy dissipation and to add into the lifespan of the network. LEACH has certain disadvantages. It is not convenient for large-sized networks. No constraints have been set for the sensor nodes' spreading manner across the network and no constraints on the level of energy. Fig 3 depicts the functioning of LEACH protocol.

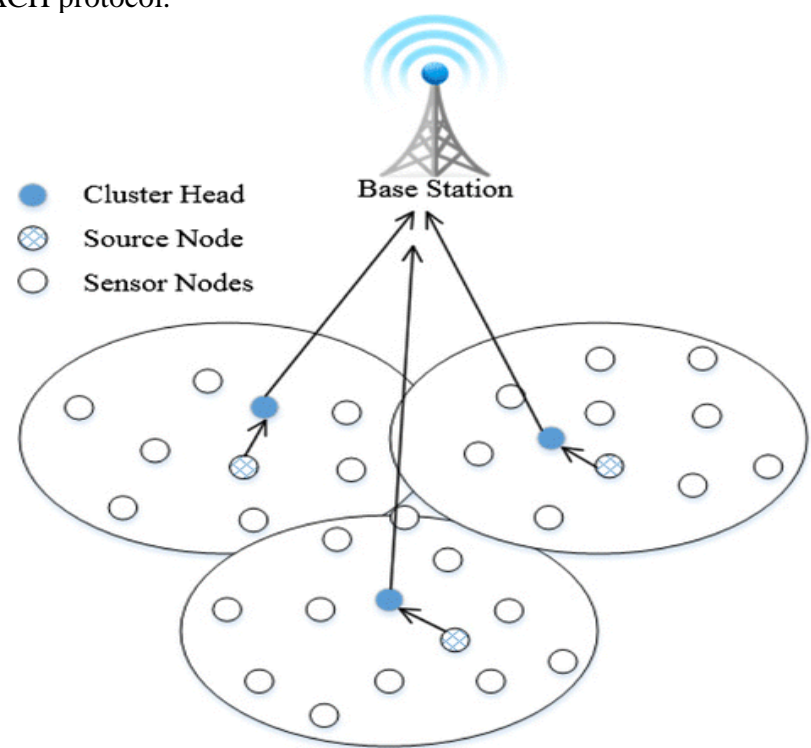

Fig. 3: Functioning of LEACH protocol [17]

\subsection{PEGASIS (Power-Efficient Gathering in Sensor Information Systems)}

It follows a chained structure in WSNs. The chain structure is formed with the help of greedy algorithm. The construction of the chain starts from that node which is in the farthest proximity in the network and then moving towards the nearest neighbors till the last node in the network. From this constructed chain, a chain leader is selected primary function will be to cumulate data and sending it to BS. The role of chain leader can be shifted. The chain leader transmits a token to end nodes at both left and right sides of the chain. When the token is received by the end node, it transmits the data towards the direction from where it acquired the token (towards the leader). Every node in the chain aggregates the data and sends to its neighbor in the chain. The process keeps going till it reaches the chain leader. The chain leader then aggregates the data and sends it to the base station. Fig 4 depicts the functioning of PEGASIS protocol. In the displayed figure, the $\mathrm{c} 2$ node is the chain leader. $\mathrm{c} 2$ will transmit the token towards $\mathrm{c} 0$ using all the nodes in between. After $\mathrm{c} 0$ receives the token, it will transmit the data in the direction of the leader. The intermediate nodes perform the action of aggregating the data till it reaches the chain leader (c2). In the given example, $\mathrm{c} 1$ performs the aggregation of its own data with $\mathrm{c} 0$ 's data and then transmits to $\mathrm{c} 2$. $\mathrm{c} 3$ performs the aggregation of self data with the data of $\mathrm{c} 4$ and conclusively sends data to $\mathrm{c} 2$. 


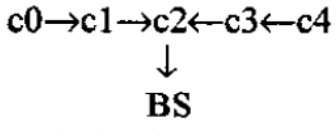

Fig. 4: Functioning of PEGASIS protocol [2]

\subsection{SPIN (Sensor Protocols for Information via Negotiation)}

It uses attribute-based naming. The interests are advertised throughout the network and the receiver wishes to get the data in which it is interested. It can send request to get the interested data. Meta data descriptors are used for this purpose. This reduces the possibility of traffic implosion and as the data is send to only those nodes which are interested in it and also the redundant data can be avoided. Here ADV packet is used for disseminating the data and REQ packet is used to inquire the data.DATA represents data. Fig 5 depicts the functioning of SPIN protocol. In the following figure, sensor A transmits an advertisement to the sensor in its close proximity. The ADV comprise the metadata which describes the data. Sensor node B is interested to have the advertised data, so it will send a request to sensor $A$ to obtain the data which it is interested to have. Further, sensor B, now advertises this data to its current neighbors. From the five neighbors of B, only four are interested to obtain that data. The main strength of SPIN in negotiation and resource adaptation as well as it reduces the consumption of energy. The disadvantages comprise that it may not be good for applications that require high reliability. Interest in more many events can lead to energy depletion.

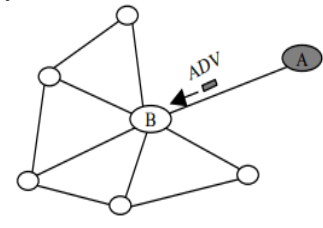

(a)

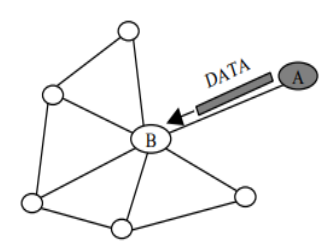

(c)

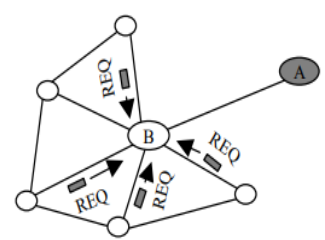

Fig. 5: Functioning of SPIN protocol [1]

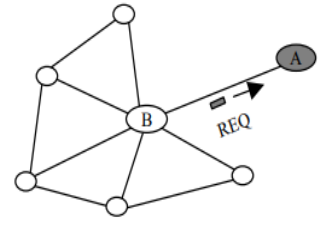

(b)

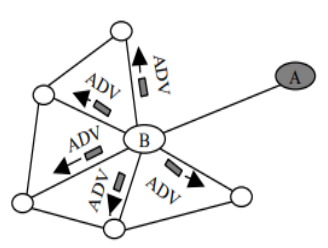

(d)

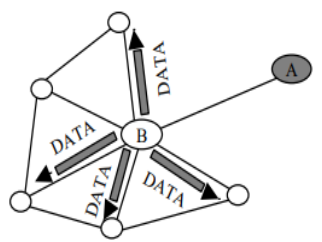

\subsection{Flooding}

Here, the packets are propagated to every single bystander node. All feasible routes are taken to be followed. Conclusively, the packet is achieved at the destination.Fig 6 depicts the scenario of flooding in WSNs. A count field is allowed so that packet is not circulated infinitely in the network. The hop sequence will be reduced by one after every hop. This protocol does not preserve tables for routing, and henceforth, is reactive in nature. But due to this nature energy dissipation is quite higher which is disadvantage. So, it has both pros and cons. As an advantage it also includes reduced cost for maintenance its simple implementation.The disadvantages of flooding cover traffic implosion, overlapping, and resource blindness. Implosion induces the network to transmit replicated packets to the same nodes. Replicated data packets will go on propagating throughput the network and therefore the sensors start obtaining replicated packets. Fig 7depicts the scenario of traffic implosion due to flooding. And in case of overlapping problem, the neighbors start obtaining replicated packets when two sensors sense the same area and they transmit that same data at the same timestamp.Fig 8 depicts the scenario of overlapping. The last one includes resource blindness, means it does not takes into account the sensor nodes' energy restraints which will induce added energy dissipation within network and therefore will reduce network life span.

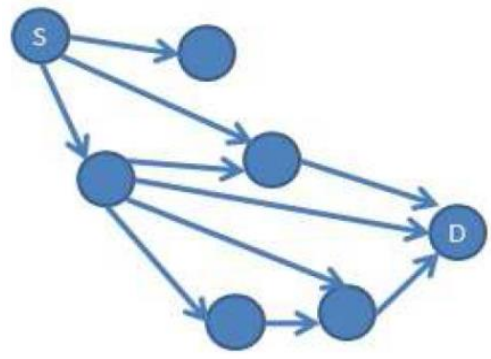

Fig. 6: Functioning of Flooding protocol [20]

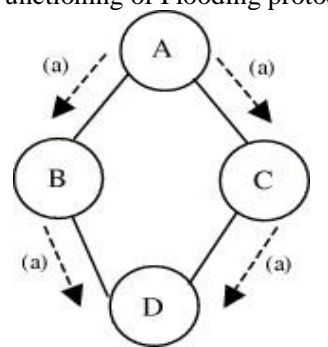

Fig 7: Traffic Implosion problem in Flooding protocol [4]

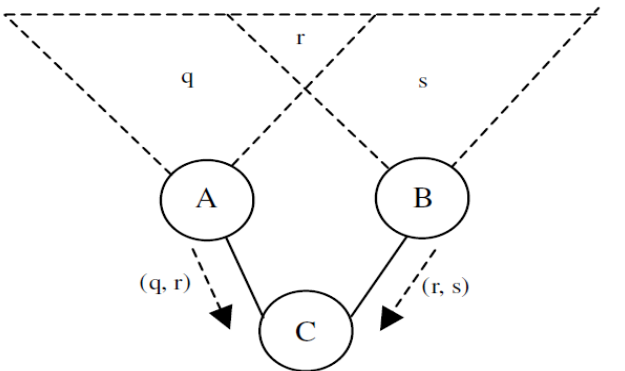

Fig 8: Overlapping problem in Flooding protocol [4]

\section{Conclusion}

This paper specifically focuses on hierarchical and data centric protocols in WSNs. A literature survey has been presented on the categories such as hierarchical, data-centric, location and flat based protocols in WSNs. Each of these categories has further types which have been elaborated in detail in this paper. LEACH protocol has hierarchical clustering as the basis and has been through enhancements on the basis of threshold, network duration, remaining energy, total number of sinks, multi-hop communication, etc.Enhancements in PEGASIS protocol have been implemented on the basis of leader selection criteria like distance between the chosen leader and other nodes, combination with LEACH protocol, remaining energy, distance among the nodes. SPIN protocol has been enhanced on the basis ofdistance discovery and acknowledgement from sink nodes.Many of these have been through various improvements.All these hierarchical and data centric protocols have overcome conventional routing protocols such as flooding and gossiping. In fact, each and every protocol has certain advantages and disadvantages of their own. Now the question ensues when to use which protocol. The choice of protocol entirely depends on what kind of application we want to use, or what kind of network our application is going to use, is location necessary for our application or not, etc. Another question ensues as to what kind of enhancements can be done further in any of the 
above said protocols. The enhancements must take into consideration the various parameters so that various comparisons can be made based upon that. These enhancements must make a big impact on the performance of these protocols to improve them in order make different applications useful for the society in a better way.

\section{References}

[1]. W. R. Heinzelman, J. Kulik, H. Balakrishnan, “Adaptive protocols for information dissemination in wireless sensor networks", Proceeding MobiCom '99 Proceedings of the 5th annual ACM/IEEE international conference on Mobile computing and networking, pp 174-185, $1999 . \quad$ [spin]

[2]. Y. Yu, R. Govindan, D. Estrin, "Geographical and Energy Aware Routing : a recursive data dissemination protocol for wireless sensor networks", 2001

[3]. S. Lindesey, C. S. Raghavendra, "PEGASIS: Power-Efficient Gathering in Sensor Information Systems", Proc. Aerospace IEEE Conference, Vol.3, pp. 3-1125 - 3-1130, 2002.

[4]. K. Akkaya, M. Younis, "A survey on routing protocols for wireless sensor networks", 2005 [implosion+overlapping]

[5]. K. Fodor, A. Vidacs, "Efficient routing to mobile sinks in wireless sensor networks", 2nd ACM International Workshop on Performance Control in Wireless Sensor Networks, 2007. [scenario of wsn]

[6]. J. Hong, J. Kook, S. Lee, D. Kwon, S. Yi, "T-LEACH: The method of threshold-based cluster head replacement for wireless sensor networks", Information Systems Frontiers, pp 513-521, 2009.

[7]. H. M. Abdulsalam, L. K. Kamel, "W-LEACH: Weighted Low Energy Adaptive Clustering Hierarchy Aggregation Algorithm for Data Streams in Wireless Sensor Networks", IEEE International Conference on Data Mining Workshops, 2010.

[8]. Z. Rehena, S. Roy, N. Mukherjee, "A Modified SPIN for Wireless Sensor Networks", Communication Systems and Networks (COMSNETS) Conference, 2011.

[9]. S. Zhang, H. Zhang, "A Review of Wireless Sensor Networks and Its Applications", International Conference on Automation and Logistics, 2012.

[10]. Q. Liao, H. Zhu, “An Energy Balanced Clustering Algorithm Based on LEACH Protocol", Proceedings of the 2nd International Conference On Systems Engineering and Modeling (ICSEM-13), 2013.

[11]. Z. Beiranvand, A. Patooghy, M. Fazeli, "I-LEACH: An Efficient Routing Algorithm to Improve Performance \& to Reduce Energy Consumption in Wireless Sensor Networks", 5th Conference on Information and Knowledge Technology (IKT), 2013.

[12]. S. E. Khediri, N. Nasri, A. Wei, A. Kachouri, "A New Approach for Clustering in Wireless Sensors Networks Based on LEACH", International Workshop on Wireless Networks and Energy Saving Techniques (WNTEST), 2014.

[13]. J.Grover, B. Noble, I.N. Sneddon, "Reliable SPIN in Wireless Sensor Networ", Reliability, Infocom Technologies and Optimization (ICRITO) (Trends and Future Directions), 3rd International Conference, 2014.

[14]. A. K. Mishra, R. U. Rahman, R. Bharadwaj, R. Sharma, "An Enhancement of PEGASIS Protocol with Improved Network Lifetime for Wireless Sensor Networks", Power, Communication and Information Technology Conference (PCITC), IEEE, 2015.

[15]. A. Tripathi, N. Yadav, R. Dadhich, "SPIN With Cluster for Data Centric Wireless Sensor Networks", Advanced Computing \& Communication Technologies (ACCT), 2015 Fifth International Conference on, 2015.

[16]. K. Khan, M. Sajid, S. Mahmood, Z. A. Khan, U. Qasim, N. Javaid, "(LEACH $)^{2}$ : Combining LEACH with Linearly Enhanced Approach for Cluster Handling in WSNs", Advanced Information Networking and Applications (AINA), IEEE 29th International Conference, 2015.

[17]. G. S. Arumugam, and T. Ponnuchamy, "EE-LEACH: development of energy-efficient LEACH Protocol for data gathering in WSN", EURASIP Journal on Wireless Communications and Networking, Springer, 2015. [Leach]

[18]. A. Razaque, M. Abdulgader, C. Joshi, "P-LEACH: Energy Efficient Routing Protocol for Wireless Sensor Networks", Systems, Applications and Technology Conference (LISAT), IEEE, 2016.
[19]. A. S. Toor, A.K. Jain, "A Survey of Routing Protocols in Wireless Sensor", IEEE International Conference on Recent Advances and Innovations in Engineering (ICRAIE), 2016.

[20]. H. ECHOUKAIRI, K. BOURGBA, M. OUZZIF, “A Survey on Flat Routing Protocols in Wireless Sensor Networks", Advances in Ubiquitous Networking, Springer", pp 311-324, 2016. [flooding]

[21]. I. Boulhares, M. Omari, "Hybridizing PEGASIS with LEACH-1R Protocols in Wireless Sensor Networks", 8th International Conference on Modelling, Identification and Control, 2016.

[22]. M. Arioua,Y. Assari, I. Ez-zazi, A. Oualkadi, "Multi-hop cluster based routing approach for wireless sensor networks", 7th International Conference on Ambient Systems, Networks and Technologies, 2016.

[23]. R. Dutta, S. Gupta, "Energy Aware Modified PEGASIS through Packet Transmission in Wireless Sensor Network", Parallel, Distributed and Grid Computing (PDGC), Fourth International Conference, 2016.

[24]. A. Krishnakumar, V. Anuratha, "An Energy-Efficient Cluster Head Selection of LEACH Protocol for Wireless Sensor Networks", Nextgen Electronic Technologies: Silicon to Software (ICNETS2), International Conference, 2017. 